\title{
Neuerscheinungen der persönlichen Mitglieder des Netzwerks TA
}

Knud Böhle, Institut für Technikfolgenabschätzung und Systemanalyse (ITAS), Karlsruhe

Mit Verweis auf den Ort, an dem die AutorInnen tätig sind, findet man auf der Karte Hinweise auf neuere Buchpublikationen, die von persönlichen Mitgliedern des Netzwerks TA stammen (diese sind jeweils fett gestellt). Die Basis für diese Auswahl sind die monatlich erscheinenden Ausgaben des openTANeuerscheinungsdienstes $1 / 2015$ bis $1 / 2016$.
Unter den insgesamt 557 TA-relevanten Neuerscheinungen aus diesem Zeitraum fanden sich 20 Buchpublikationen persönlicher NTA-Mitglieder. Ihre Zusammenstellung, ein kleines Schaufenster der NTA-Produktivität, soll die Leserinnen und Leser der TATuP auf interessante Neuerscheinungen der TA-Community aufmerksam machen.

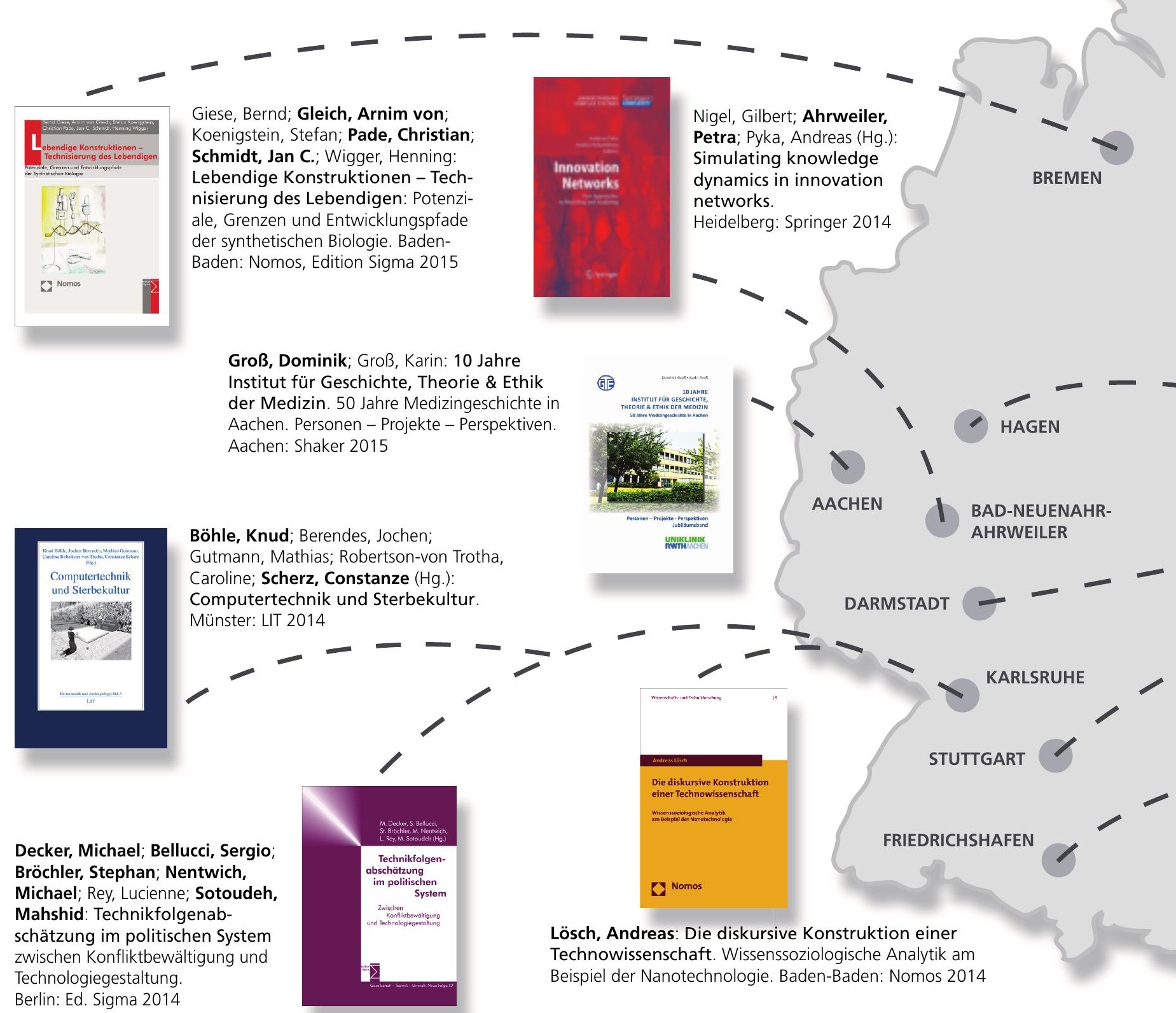



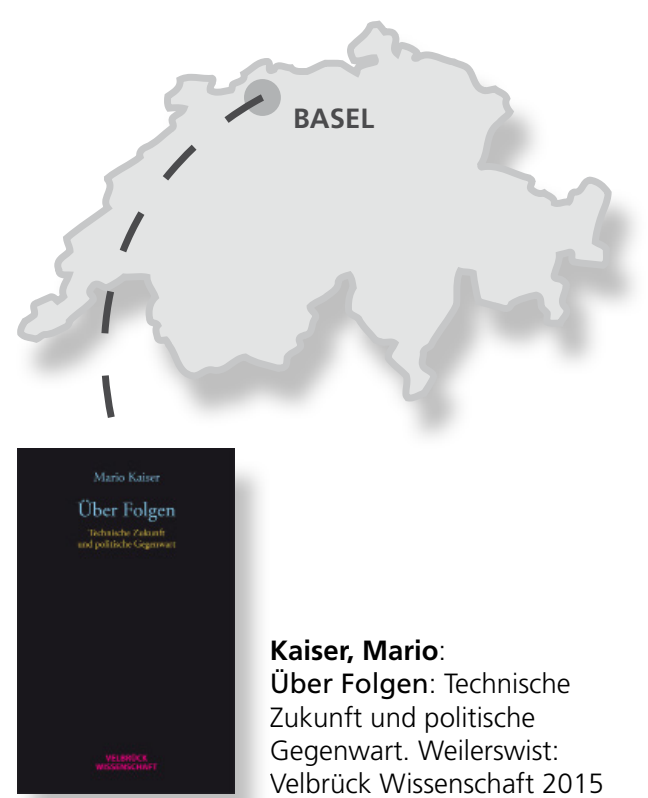

Kaiser, Mario:

Über Folgen: Technische

Zukunft und politische

Gegenwart. Weilerswist:

Velbrück Wissenschaft 2015

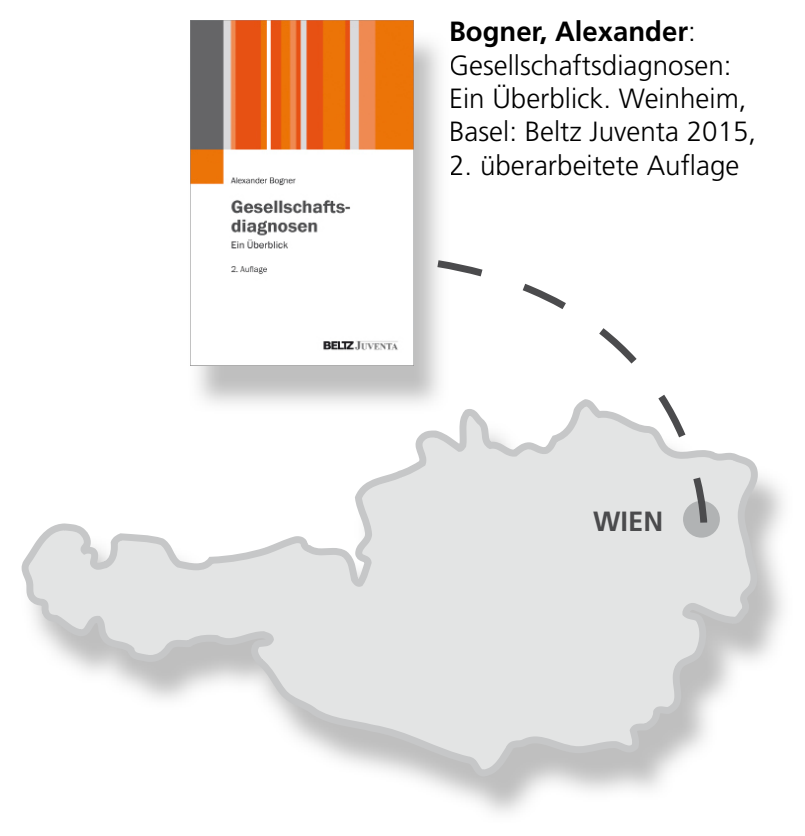

\section{Neuerscheinungen des NTA als TATuP-Rubrik?}

Der openTA-Neuerscheinungsdienst basiert auf einem monatlich ausgeführten Suchprofil in der Deutschen Nationalbibliografie (vgl. zum Neuerscheinungsdienst Böhle, $\mathrm{K}$. et al.: openTA-Neuerscheinungsdienst "ueberdenTAellerrand" - Konzept und Realisierung. openTA-Dokument Nr. 6. Karlsruhe: KIT-ITAS 2015). Eine hundertprozentige Abdeckung der Buchpublikationen der persönlichen NTAMitglieder, die in der Deutschen Nationalbibliografie erfasst sind, ist allerdings auf diesem Weg nicht zu erreichen. Dazu müsste über die Namen der persönlichen Mitglieder oder noch besser über die eindeutige, standardisierte Identifikationsnummer für Personen gesucht werden. Daraus könnte dann ein neuer openTA-Dienst entstehen: Neuerscheinungen der persönlichen NTA-Mitglieder aus der deutschen Nationalbibliografie. Das openTA- Portal fungierte als Schaufenster für diese Neuerscheinungen. Die persönliche Mitgliedschaft im NTA führte dann dazu, dass die eigenen Buchpublikationen über das openTA-Portal und auch - wie hier vorgeschlagen - über eine neue Rubrik der TATuP beworben würden.
Dieses Angebot bliebe aber unvollständig ohne die Anlieferung bibliografischer Daten über Neuerscheinungen seitens der institutionellen NTA-Mitglieder. Aus beiden Aktivitäten zusammen, der automatisierten Suche nach Neuerscheinungen von NTA-Mitgliedern in der Deutschen Nationalbibliografie und der Meldung von Neuerscheinungen durch die NTA-Einrichtungen, ließe sich eine wirklich attraktive TATuP-Rubrik mit aktuellen Hinweisen auf neue (N)TA-Literatur entwickeln. Diesen Vorschlag stellen wir hiermit zur Diskussion.

Dieser TATuP-Labor-Artikel erscheint in leicht modifizierter Form auch als Blog-Beitrag im openTA-Blog, und kann dort online kommentiert werden. "Neuerscheinungen aus dem NTA" ist der dritte Beitrag für das TATuP-Labor. Das TATuP-Labor ist unser Experimentalkasten für den im Zuge des 25-jährigen Bestehens der TATuP anstehenden Relaunch der Zeitschrift. Was halten Sie von einer solchen neuen Rubrik? Und wie gefällt Ihnen die typographische Gestaltung? Teilen Sie uns Ihre Meinung mit, damit wir TATuP noch besser auf Ihre Bedürfnisse ausrichten können! redaktion@tatup-journal.de 